EPJ Web of Conferences 32, 04020 (2012)

DOI: 10.1051/epjconf/20123204020

(C) Owned by the authors, published by EDP Sciences, 2012

\title{
Progress on corrugated waveguide components suitable for ITER ECH\&CD transmission lines
}

\author{
R.A. Olstad, R.W. Callis, M. Cengher, J.L. Doane, Y.A. Gorelov, H.J. Grunloh, C.P. Moeller \\ and C.J. Murphy
}

General Atomics, P.O. Box 85608, San Diego, California 92186-5608, USA

\begin{abstract}
The $2 \mathrm{MW} \mathrm{cw}$ requirement for ITER EC transmission lines has led GA to design a number of new and modified components, namely power monitors and polarizers to meet this requirement. In addition, the ITER transmission lines may require sliding joints or bellows to accommodate the thermal expansion of the lines and/or vessel motion. The ex-vessel sections of the EC launchers require double seal waveguides, miter bends and possibly double seal sliding joints to assure tritium retention in this region. GA has developed designs for many of these components and some of them have already been tested at high power at the JAEA RF Test Stand. Thermal analyses of the standard GA power monitor miter bend show that our standard design is suitable for $1 \mathrm{MW} \mathrm{cw}$ operation when the $\mathrm{H}$-field is in the plane of the miter bend. For $2 \mathrm{MW} \mathrm{cw}$ operation a modified design, which also requires $\mathrm{H}$-plane orientation, has been developed and a prototype has been fabricated and is ready for testing. For long pulse/cw operation, a 2 MW calorimetric miter bend with thermally isolated mirror has been designed and a prototype has been fabricated. Since the mirror is thermally isolated, calorimetry on the mirror cooling water can provide a measure of absorbed power. Such a miter bend, when made in a double seal version, could be useful for monitoring total power at the end of an EC transmission line just before the in-vessel section of an EC launcher. A mode analyzer using an all metal water-cooled beam splitter is being developed for use in measuring in real time the $\mathrm{HE}_{11}$ and higher order mode content or total power in EC transmission lines. Such a high power diagnostic can be very useful in optimizing the alignment of the MOU output into a transmission line and in monitoring the $\mathrm{HE}_{11}$ mode purity at various locations in a transmission line. Prior to making a $63.5 \mathrm{~mm} 170 \mathrm{GHz}$ version suitable for use on ITER, several prototype $31.75 \mathrm{~mm} 110 \mathrm{GHz}$ versions have been fabricated and tested at DIII-D. A $170 \mathrm{GHz}$ version is now being fabricated. This paper addresses the performance characteristics, design features and test results for the new and modified components being developed to assure low loss transmission and acceptable component stresses for $2 \mathrm{MW}$ cw opération.
\end{abstract}

This is an Open Access article distributed under the terms of the Creative Commons Attribution License 2.0, which permits unrestricted use, distribution, and reproduction in any medium, provided the original work is properly cited. 


\section{Introduction}

A thorough description of the EC H\&D transmission lines for ITER has been given in Ref. [1]. In order to meet the ITER requirements of having each of the 24 main transmission lines and 32 additional lines to the upper launchers be capable of $2 \mathrm{MW} \mathrm{cW}$ operation, we have designed and/or modified component designs to increase their performance from the typical $1 \mathrm{MW} \mathrm{cW}$ required prior to ITER. To meet this $2 \mathrm{MW} \mathrm{cw}$ performance we have developed a new design for the power monitor miter bend and are in the process of increasing the power capability of polarizer miter bends. We have also developed a new component called a calorimetric miter bend that is suitable for monitoring power over long time scales based on monitoring the ohmic power dissipated in a copper miter bend mirror. Our dc break design was modified so that cooling water is not in contact with aluminum, and we are developing a mode analyzer to monitor the modes present in the corrugated waveguide transmission lines wherever a miter bend can be inserted into the line.

The sections of the transmission lines between the CVD diamond barrier window and the invessel equatorial and upper launchers have special design requirements to assure retention of the tritium that will be present during DT operation. The concept decided upon by ITER-IO is to use double Helicoflex ${ }^{\circledR}$ seals at all locations where there could be a path between vacuum and the external environment. We have designed such components and are presently fabricating prototype components for ITER-IO.

For all of the transmission line components, tight dimensional tolerances must be met in order to minimize mode conversion losses due to offsets and tilts between components and due to waveguide curvature. These demands are driven by the need to achieve $>83 \%$ transmission efficiency so that $>20 \mathrm{MW}$ of $\mathrm{HE}_{11}$ power is launched into the tokamak from $24 \mathrm{MW}$ at the MOU outputs.

Progress in each of the above areas is presented in this paper.

\section{Power monitor miter bends}

GA's standard $1 \mathrm{MW}$ power monitor bend uses a linear array of coupling holes near the center the mirror to couple power incident on the mirror to pickup horns on the backside of the miter bend. A fused silica wedge inserted and sealed into the backside of the mirror is used to direct forward and reverse power to pickup horns to monitor power in the forward and reverse directions. The Glidcop® or chromium copper thickness in the coupling hole region must be thin so that there is adequate signal strength. The thinness of the copper limits the ability to remove ohmic heat deposited in the vicinity of the coupling holes. For $1 \mathrm{MW} \mathrm{cw}$ incident in $\mathrm{H}$-plane polarization (electric field is perpendicular to the plane of the miter bend), the calculated maximum surface temperature of the copper mirror for $850 \mathrm{~W}$ absorbed at room temperature is $72^{\circ} \mathrm{C}$, and the maximum stress is less than $255 \mathrm{MPa}$, which is below the $300 \mathrm{MPa}$ yield stress for the mirror materials. For $1 \mathrm{MW}$ incident in E-plane polarization or $2 \mathrm{MW}$ in H-plane polarization, calculated stresses exceed the yield stress.

To increase the power handling ability of the power monitor miter bend to $2 \mathrm{MW} \mathrm{cw}$, a new design was developed [2]. In this design, an array of small cutoff holes in the mirror radiates power to a small rectangular waveguide parallel to the mirror surface. The rectangular waveguide phase is matched to the incident high power wave at $45^{\circ}$ to the mirror surface. Finite element thermal and stress analysis shows that for $2 \mathrm{MW}$ incident $(1700 \mathrm{~W}$ absorbed at room temperature for $\mathrm{H}$-plane polarization) the maximum copper temperature is $46^{\circ} \mathrm{C}$ and the maximum stress is $91 \mathrm{MPa}$. This is well under the $300 \mathrm{MPa}$ yield stress for chromium copper alloy, so we conclude that this new power monitor is suitable for $2 \mathrm{MW} \mathrm{cw}$ operation for $\mathrm{H}$-plane incidence.

A prototype of this new power monitor design has been fabricated, as shown in Figure 1. Low power tests showed a coupling of $-68 \mathrm{~dB}$. Plans for performing high power tests at the JAEA RF Test Stand are being made. 


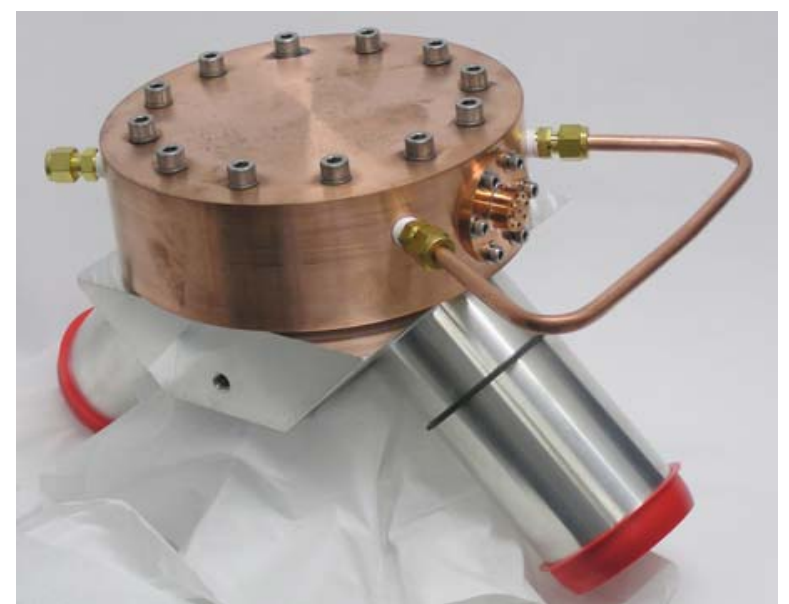

Fig. 1. Prototype $2 \mathrm{MW} 170 \mathrm{GHz}$ Power Monitor Miter Bend.

\section{Calorimetric miter bend}

We have designed and built a calorimetric miter bend that can be used for any incident polarization, in contrast with our $2 \mathrm{MW}$ power monitor miter bend which requires H-plane polarization of the incident beam [2]. The mirror in this miter bend is thermally isolated so that cooling water calorimetry can provide a measure of absorbed power. The calorimetry measurements need to be calibrated against incident power and polarization because the ohmic losses depend on the temperature of the mirror surface and on the incident polarization. Such a component would be useful for monitoring overall power levels at various locations along a transmission line for arbitrary polarizations.

A prototype of this miter bend has been fabricated (Figure 2) and plans are being made to test it at the JAEA RF Test Stand. A version using double Helicoflex seals could be designed so that it could be used in the ex-vessel portion of the transmission line.

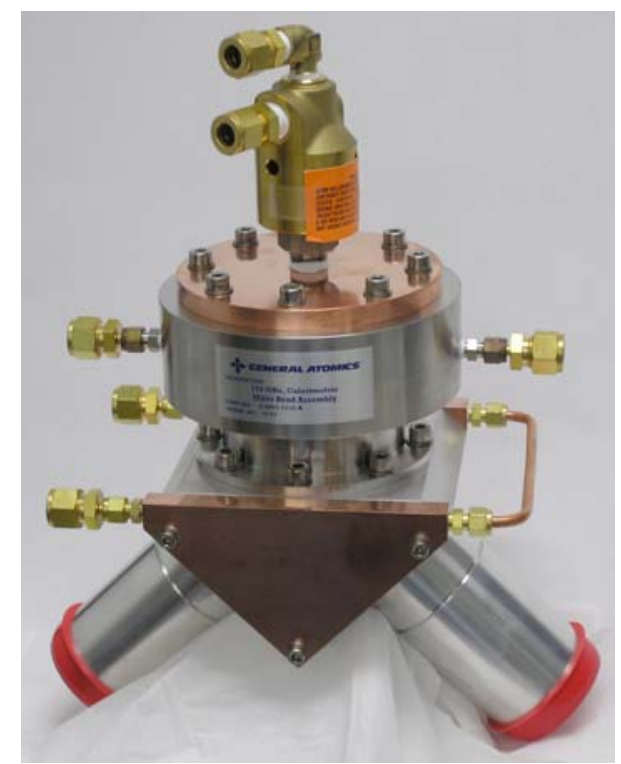

Fig. 2. Prototype $2 \mathrm{MW} 170 \mathrm{GHz}$ Calorimetric Miter Bend. 


\section{MW dc break}

A prototype dc break with aluminum alloy flanged waveguides was successfully tested at the JAEA RF Test Stand [3]. These tests showed that the température increase of the outer surface of the ceramic insulator was about $8^{\circ} \mathrm{C}$ vs a safe operating limit of about $50^{\circ} \mathrm{C}$. This was equivalent to $27^{\circ} \mathrm{C}$ for $2 \mathrm{MW}$ transmssion. Becasue of the presence of higher order modes, this increase was larger than would have been the case with pure $\mathrm{HE}_{11}$ transmission. For pure $\mathrm{HE}_{11}$, the calculated temperature increase is about $10^{\circ} \mathrm{C}$ for $2 \mathrm{MW} \mathrm{cw}$ transmission when the adjacent waveguide stubs are water-cooled. Since it was shown that the DC break is suitable for $2 \mathrm{MW}$ operation, a new prototype was fabricated using chromium copper flanged waveguides. These waveguides stubs can be cooled with water since direct contact between water and copper alloy is permitted by ITER, but direct contact with aluminum is not. The USIPO will be testing the dc break at their test stand or possibly at the JAEA RF Test Stand.

\section{Polarizer miter bends}

In tests of a pair of polarizer miter bends at the JAEA RF Test Stand in 2008 and 2010, it was found from calorimetry on the polarization rotator mirror that, at large rotation angles, the ohmic losses were approximately 2.5 times the values expected from the theory developed by J. Doane [3, 4]. Our most recent design has more radial cooling within the mirrors than was the case with the mirrors tested at JAEA. With this enhanced cooling design, the polarizers appear suitable for $1 \mathrm{MW} \mathrm{cW}$ operation for any mirror rotation angle and polarization. Use at $2 \mathrm{MW} \mathrm{cW}$ operation requires the use of optimized rotation angles for the lowest ohmic loss solutions and that the first polarizer miter bend be the polarization rotator in an H-plane orientation. J. Doane is also developing an FDTD model of the polarizer mirrors and plans to benchmark model results against room temperature measurements obtained by W. Kasparek's group at the University of Stuttgart [5].

For $2 \mathrm{MW} \mathrm{cw}$ operation for any solutions of mirror angles, reduced losses at the mirror surface will be required. The mirrors originally fabricated had grooves that were made using a wire EDM technique. Optical examination showed that the surfaces were quite rough because of the re-cast layer. The mirror surfaces were re-machined, but there were still visible machining lines parallel to the axis of the grooves, and re-testing them did not show reduced losses. It was hypothesized that well-polished mirror surfaces will be needed to reduce losses on these mirrors. GA fabricated two new sets of polarizer mirrors, one set with well-machined surfaces and another set that was polished after machining. Both sets of mirrors were provided to MIT for low power testing at their Plasma Science and Fusion Center. The experimental setup and initial tests are being performed now. Based on results from these tests, new mirrors will be fabricated for the polarizer miter bends at JAEA so that additional high power tests can be performed.

\section{$6170 \mathrm{GHz}$ mode analyzer}

A mode analyzer using an all metal water-cooled beam splitter is being developed for use in measuring in real time the $\mathrm{HE}_{11}$ content of the beam propagating in a transmission line. The beam splitter is mounted between two back to back miter bends and both sides of the mirror are evacuated. A small fraction of the forward power is coupled though an array of small coupling holes in the mirror. Though incorporation of an appropriate taper and directional couplers on the low power side of the mirror, the fraction of power in $\mathrm{HE}_{11}, \mathrm{HE}_{21}$ and $\mathrm{TE}_{01}$, for example, can be measured. These latter two modes are generated when there is an offset or tilt at the entry to the waveguide. A nonoptimum beam radius will generate higher-order $\mathrm{HE}_{1 \mathrm{n}}$ modes. A prototype mode analyzer was made in $31.75 \mathrm{~mm}$ waveguide for $1 \mathrm{MW} 110 \mathrm{GHz}$ operation and was successfully tested at DIII-D [6]. These tests confirmed that the best alignment corresponds to the maximum $\mathrm{HE}_{11}$ signal and minimum $\mathrm{HE}_{21}$ and $\mathrm{TE}_{01}$ signals. 
A $63.5 \mathrm{~mm}$ diameter version for operation at $2 \mathrm{MW} 170 \mathrm{GHz}$ is being fabricated. Whereas the smaller $110 \mathrm{GHz}$ version had only 840 coupling holes, the $170 \mathrm{GHz}$ version has about 5000 coupling holes. The coupling holes are drilled into water-cooled posts machined into the Glidcop mirror. Before the coupling holes are drilled, a Glidcop screen and braze alloy screen are secured to the posts by deforming the ends of the posts into tiny rivets. After the heads are deformed into rivets, the assembly is brazed. The large number of posts requires an automated technique to perform the riveting operation, and this too is being developed. When completed, hopefully this prototype mode analyzer can be tested at either the JAEA RF Test Stand or the USIPO ITER Transmission Line Test Stand [7].

\section{ITER double seal ex-vessel launcher components}

Tritium retention concerns for components in the region between the ITER in-vessel launcher and the diamond barrier window led GA to develop designs for various transmission line components using double Helicoflex ${ }^{\circledR}$ seals (DHS) at joints between adjacent components [2]. These double seals provide a high level of assurance that tritium in this section of the transmission line will not leak into the port cell if a seal should fail.

Based on thèse designs, GA is fabricating a number of such prototype components for testing at the JAEA RF Test Stand. These include DHS straight waveguides, DHS miter bends $\left(90^{\circ}\right.$ and non$90^{\circ}$ ), DHS bellows/sliding waveguide joint, and various adapter sections between DHS components and those using single Helicoflex ${ }^{\circledR}$ seals or Conflat flanges at interfaces.

At the connecting ends of DHS components, there is a shallow plenum between the two seals so that this space can be monitored for the presence of tritium. Connecting fittings also provide a metal seal so that tritium cannot be released into the port cell. Similarly, miter bend mirrors are installed using two Helicoflex ${ }^{\circledR}$ seals with a monitored plenum between them. The waveguide sliding joint is based on a single seal version successfully tested at JAEA [2]. The DHS version uses two concentric stainless steel welded bellows, and provision is made to monitor the space between them.

\section{Transmission line efficiency}

Much work has gone into calculating the transmission line efficiency expected for ITER transmission lines. The ITER goal is to inject $20 \mathrm{MW}$ of ECH power into the tokamak with total output from the gyrotron MOUs of $24 \mathrm{MW}$, equivalent to an $83 \%$ transmission efficiency. F. Gandini et al [1] show that a transmission efficiency of $90 \%-92 \%$ should be achievable and that is therefore the efficiency requested by ITER. The $\mathrm{HE}_{11}$ mode purity of the mm-wave beam entering the launcher must be high since modes other than $\mathrm{HE}_{11}$ will not be focused at the desired location in the vessel. A detailed analysis of transmission line efficiency is beyond the scope of this paper. However, J. Doane assessed ohmic and mode conversion losses in various components, including the impact of component fabrication tolerances, and also addressed mode conversion losses due to waveguide sag and waveguide support spacing [8]. M. Shapiro addressed the impact of $\mathrm{HE}_{11}$ mode purity at the entrance to the waveguide on mode conversion losses [9]. The general consensus is that high transmission efficiency is possible but will be very demanding on the precision that must be achieved on individual components and in the installation of the components. Effects from thermal expansion of the lines, even with waveguide cooling, will have to be taken into account. The ITER Transmission Line Test Stand being built at Oak Ridge National Laboratory should provide valuable experimental data on transmission line efficiency to be expected at ITER. 


\section{Conclusions}

Excellent progress is being made in developing new component designs and modifying existing designs to meet the challenge of high transmission line efficiency and the thermal management demands of $2 \mathrm{MW} \mathrm{cw} \mathrm{operation.} \mathrm{While} \mathrm{final} \mathrm{specifications} \mathrm{for} \mathrm{ITER} \mathrm{transmission} \mathrm{line} \mathrm{components}$ have not yet been determined, operation at $1 \mathrm{MW} \mathrm{cw}$ does not appear to be a problem, and operation at levels up to $2 \mathrm{MW} \mathrm{cw}$ appears to be feasible. It remains critically important to test components at the high power, long pulse conditions the components will encounter on ITER to validate designs and to determine where improved designs may be needed. The planned high power testing of components at JAEA and ORNL will provide valuable information to assure that components are designed to satisfy the ITER performance requirements.

\section{Acknowledgment}

This work supported by US Department of Energy under DE-FC02-04ER54698 and GA internal funding. We also gratefully acknowledge Dr. Keishi Sakamoto and staff at JAEA in testing these components at high power levels under a US/Japan RF Heating Technology Exchange. In addition we would like to thank Dr. Richard Temkin and associates at the MIT Plasma Science and Fusion Center for their efforts in performing low power measurements on polarizer mirrors.

\section{References}

1. F. Gandini et al, "The EC H\&CD Transmission Line for ITER," J. Infrared, Millimeter and THz Waves 32, 358 (2011).

2. R.A. Olstad et al, "Designs of New Components for ITER ECH\&CD Transmission Line," Proc. $35^{\text {th }}$ Int. Conf. on Infrared Millimeter and Terahertz Waves (IRMMW-THz), 2010.

3. R.A. Olstad et al, "Design and High Power Testing of ITER ECH\&CD Transmission Line Components," Proc. $34^{\text {th }}$ Int. Conf. on Infrared Millimeter and Terahertz Waves (IRMMW-THz), 2009.

4. R.W. Callis et al. "Testing of ITER-Class ECH Transmission Line Components at the JAEA Radio-Frequency Test Stand," Proc. 23 ${ }^{\text {rd }}$ IAEA Fusion Energy Conf., Republic of Korea, 2010.

5. W. Kasparek, A. Fernandez, F. Hollman, and R. Wacker, "Measurements Ohmic Losses of Metallic Reflectors at $140 \mathrm{GHz}$ Using a 3-Mirror Resonator Technique," Int. J. Infrared Millimeter Waves 22, 1695 (2001).

6. M. Cengher et al, "Transmission Lines Power Measurements for the $110 \mathrm{GHz}$ Electron Cyclotron Heating System and Gyrotron Operational Performance," Proc. 35 ${ }^{\text {th }}$ Int. Conf, on Infrared Millimeter and Terahertz Waves (IRMMW-THz), 2010

7. D.A. Rasmussen et al. "Design of the ITER Electron Cyclotron Heating and Current Drive Waveguide Transmission Line," Proc. 4th IAEA TM on ECRH Physics and Technology for ITER, Vienna, Austria, 2007.

8. J. Doane, "Design of Circular Corrugated Waveguides to Transmit Millimeter Waves at ITER," Fusion Sci. Technol. 53, 159 (2008).

9. M.A. Shapiro et al, "Loss Estimate for ITER ECH Transmission Line Including Multimode Propagation," Fusion Sci. Technol. 57, 196 (2010). 